\title{
Fornix Microstructure Correlates with Recollection But Not Familiarity Memory
}

\author{
Sarah R. Rudebeck, ${ }^{1}$ Jan Scholz, ${ }^{2}$ Rebecca Millington, ${ }^{1}$ Gustavo Rohenkohl, ${ }^{1}$ Heidi Johansen-Berg, ${ }^{2}$ and Andy C. H. Lee ${ }^{1}$ \\ ${ }^{1}$ Department of Experimental Psychology, University of Oxford, Oxford OX1 3UD, United Kingdom, and ${ }^{2}$ Centre for Functional MRI of the Brain, University \\ of Oxford, Oxford OX3 9DU, United Kingdom
}

The fornix is the main tract between the medial temporal lobe (MTL) and medial diencephalon, both of which are critical for episodic memory. The precise involvement of the fornix in memory, however, has been difficult to ascertain since damage to this tract in human amnesics is invariably accompanied by atrophy to surrounding structures. We used diffusion-weighted imaging to investigate whether individual differences in fornix white matter microstructure in neurologically healthy participants were related to differences in memory as assessed by two recognition tasks. Higher microstructural integrity in the fornix tail was found to be associated with significantly better recollection memory. In contrast, there was no significant correlation between fornix microstructure and familiarity memory or performance on two non-mnemonic tasks. Our findings support the idea that there are distinct MTL- diencephalon pathways that subserve differing memory processes.

\section{Introduction}

An ongoing controversy in memory research pertains to the neural substrates of recognition memory, with recognition believed to consist of recollection (remembering contextual information) and familiarity (feeling of knowing). One dual-process theory posits that these processes are mediated by distinct medial temporal lobe (MTL) regions, with the hippocampus critical for recollection and the perirhinal cortex important for familiarity (Aggleton and Brown, 1999; Eichenbaum et al., 2007). An alternative unitary view argues that both structures support recollection and familiarity, and that memory strength is a more critical determinant of MTL involvement during recognition (Squire et al., 2007).

Although most human studies on this issue have examined the effects of MTL lesions in amnesic patients and MTL activity during recognition memory in healthy participants, a handful have investigated the role of the fornix in recognition. The fornix is the main white matter tract connecting the MTL and medial diencephalon and since it is comprised predominantly of hippocampal projections, the integrity of this tract may be critical for hippocampal-dependent processes. Although initial studies of human fornix lesions failed to report amnesia following fornix damage (Garcia-Bengochea and Friedman, 1987), later studies have consistently found memory loss, although the exact pattern of memory impairment has varied (Gaffan et al., 1991; Hodges and

\footnotetext{
Received Sept. 22, 2009; revised 0ct. 14, 2009; accepted 0ct. 24, 2009.

This research was funded by the Wellcome Trust (A.C.H.L., H.J.-B.) and Medical Research Council (J.S.). We thank all participants for their time; staff at the University of Oxford Centre for Clinical Magnetic Resonance Research for assistance with data collection; Dr. Gwenaëlle Douaud and Prof. Steve Smith for analysis advice; and Prof. David Gaffan and Dr. Anna Mitchell for helpful discussion.

Correspondence should be addressed to Sarah Rudebeck or Andy Lee, Department of Experimental Psychology, University of 0xford, South Parks Road, 0xford 0X1 3UD, UK, E-mail: sarah.rudebeck@psy.ox.ac.uk or andy.lee@ psy.ox.ac.uk.

DOI:10.1523/JNEUROSCI.4707-09.2009

Copyright $\odot 2009$ Society for Neuroscience $\quad$ 0270-6474/09/2914987-06\$15.00/0
}

Carpenter, 1991; D’Esposito et al., 1995; McMackin et al., 1995; Aggleton et al., 2000; Poreh et al., 2006; Vann et al., 2009).

Critically, studying the impact of human fornix damage can be methodologically fraught, making it almost impossible to isolate the role of the fornix in memory (Aggleton, 2008). Fornix damage is often accompanied by damage to surrounding structures, and cases of complete fornix transection are rare. Moreover, fornix volume following atrophy is difficult to measure due to distortion, perhaps explaining the failure of previous work to observe a significant correlation between fornix volume in patients with fornix damage and performance on a range of standard memory tests (Tsivilis et al., 2008).

An alternative, novel method to investigate the role of the fornix in recognition is to assess whether variations of this tract at a microstructural level in healthy individuals are related to differences in recollection and/or familiarity. This follows on from recent work that found preliminary evidence in a small group of healthy participants to hint that variations in fornix white matter microstructure may be associated with differences in episodic memory (Nestor et al., 2007).

We used diffusion-weighted imaging (DWI) to derive a measure [fractional anisotropy (FA)] of the microstructural integrity of 25 healthy individuals' fornices. To determine whether microstructure is related to specific mnemonic processes, the relationship between FA and performance on two recognition memory tasks and two non-mnemonic control tasks was investigated. Whereas a unitary view of recognition memory predicts that improved fornix microstructure may be associated with better recollection and familiarity, a dual-process view suggests that improved fornix microstructure may be correlated with better recollection only.

\section{Materials and Methods}

\section{Participants}

Twenty-five right-handed neurologically healthy volunteers (14 male; aged 22-31 years; mean $=25.30 ; \mathrm{SD}=2.89$ ) participated after giving 
written informed consent. This work received approval from the Oxfordshire Research Ethics Committee (07/H0604/115).

\section{Behavioral paradigms}

All behavioral tasks were programmed using Presentation software (Neurobehavioural Systems) and administered in a counterbalanced order across participants on a 14.1 inch laptop computer with $440 \times 900$ pixel screen resolution.

Recognition memory tasks. One scene and one object recognition task were used. The former involved 240 grayscale photographs of unfamiliar indoor and outdoor scenes, which did not contain people, objects, or words. For the object test, 240 grayscale photos of everyday objects were used. In both tests the stimuli were split into 120 items for an encoding phase and 120 foil items, which were presented with the encoding items for a test phase. In the encoding phase participants were presented with individual images and asked whether each scene was indoor or outdoor, or each object could fit in a shoebox. Following a 20 min delay, during which an unrelated distracter task was completed, the test phase took place. Participants were presented with the encoding items intermixed randomly with the foils and asked to make a recognition judgment for each item using a six-point confidence scale (" 1 " = confident an item is new; " 6 " = confident an item is old).

Receiver operating characteristic (ROC) curves for each task were derived by plotting $P$ (hits) versus $P$ (false alarms) for each consecutive confidence response level. Two separate models, the dual-process signal detection (DPSD) model (Yonelinas, 1994) and unequal variance signal detection (UVSD) model (Wixted, 2007), were fit to this data. The former assumes that recognition memory consists of independent recollection and familiarity components, whereas the latter argues that the degree of memory strength underlies recognition performance. For the DPSD model, a Microsoft Excel Solver that implements a sum-ofsquares search algorithm was used to obtain estimates of recollection (ro) and familiarity $\left(d^{\prime}\right)$ for each participant. For the UVSD model, performance was measured by two parameters: the slope of the $z$-transformed ROC data, which reflects the ratio of variance of the encoded and foil item noise distributions, and sensitivity ( $\mathrm{Da}$; calculated from the slope of the $z$-transformed ROC data and its $y$-axis intercept), which reflects overall accuracy (Macmillan and Creelman, 2004).

Control tasks. One color-discrimination and one attention task were administered. The former was a color oddity task (Barense et al., 2007) in which four colored squares $(425 \times 275$ pixels) were shown in each trial (64 total), with three being an identical color and one a different color. Participants selected the odd one out by pressing the corresponding key on a keyboard, with accuracy recorded. Luminance was equal across all four squares and each color was trial-unique.

The attention task was a motor version of the Posner cueing paradigm (Rushworth et al., 2001). On each trial, a diamond shaped cue was followed by a colored Gabor patch (target), both centrally presented. Participants were asked to respond to the target depending on the combination of color and orientation (counterbalanced) of the Gabor (lefthand button press for red/vertical and green/horizontal, right-hand button press for green/vertical and red/horizontal). This orthogonal manipulation of the target was used so that neither feature alone specified the correct response. A lightened side of the diamond cues informed participants about the type of target that would appear, and thus the required response. Cues were valid in $54 \%$, invalid in $18 \%$, and neutral in $28 \%$ of trials, with performance measured by correct response times.

\section{Imaging data acquisition, preprocessing, and analyses}

For each participant, two DWI datasets and one T1-weighted anatomical scan were acquired on a $3 \mathrm{~T}$ Trio Sonata (Siemens) with a 12-channel head coil at the University of Oxford Centre for Clinical Magnetic Resonance Research (for details, see supplemental material, available at www. jneurosci.org). DWI allows the investigation of microstructural properties of brain white matter by using the fact that water molecules diffuse quicker along the direction of an axon bundle than in the perpendicular direction. FA (Basser et al., 1994) quantifies this directional diffusion at each voxel, with values approaching 1 reflecting diffusion predominantly along one axis and values approaching 0 indicating diffusion in all direc- tions. FA is thought to reflect structural properties such as axonal diameter, density, and myelination (Beaulieu, 2002) and is taken to reflect white matter microstructure integrity.

All image preprocessing and analyses were performed using tools from the FMRIB Software Library (www.fmrib.ox.ac.uk/fsl). Three complementary approaches were used to investigate the role of the fornix in recognition memory (for details, see supplemental material, available at www.jneurosci.org): (1) tract-based spatial statistics (TBSS), (2) individual subject tractography and FA extraction, and (3) white matter voxelbased morphometry (VBM).

\section{TBSS}

TBSS allows voxelwise statistical comparison between individual subjects' FA maps and has been fully described previously (Smith et al., 2006). To investigate the relationship between FA and performance on the behavioral tasks, multiple general linear models (GLMs) were implemented: (1) an ROC DPSD model GLM, with explanatory variables of scene recollection, object recollection, scene familiarity, and object familiarity; (2) a UVSD model GLM, with explanatory variables of scene $z$-ROC slope, object $z$-ROC slope, scene sensitivity, and object sensitivity; (3) a color oddity GLM, with one explanatory variable of proportion correct; and (4) a motor attention GLM, with explanatory variables of neutral, valid, and invalid response times. Contrasts of interest were then conducted to examine and compare across explanatory variables.

Since our a priori hypotheses pertain to the fornix, we restricted our statistical analyses to this structure using a mean fornix mask (829 voxels at $1 \times 1 \times 1 \mathrm{~mm}$ ), which was created on a mean group FA map using anatomical guidelines (Bilir et al., 1998). A threshold of $p<0.005$ uncorrected, cluster size $\geq 5$ voxels was applied to identify fornix regions in which there was a significant relationship between FA and a behavioral measure of interest. This threshold is equivalent to a mapwise falsepositive rate for the fornix mask of $p<0.001$ (estimated using a Monte Carlo procedure as implemented in the AlphaSim program in the AFNI software package). Since we had no predictions beyond the fornix, a more stringent threshold of $p<0.001$ uncorrected, cluster size $\geq 10$ voxels was used to explore the rest of the brain white matter $(12,303$ voxels; equivalent false-positive rate $p<0.0001$ ). All coordinates are in Montreal Neurological Institute 152 space.

\section{Individual subject tractography and FA extraction}

An alternative approach to TBSS involved extracting the mean FA across each participant's fornix and correlating these values with the acquired behavioral measures. Multifiber probabilistic tractography (Behrens et al., 2003) was used to trace each individual's fornix and create a priori defined masks for the fornix tail (left and right combined), body, and column. Using these masks, mean FA values were extracted from individual FA maps and correlated with behavioral measures using standard statistical software.

\section{White matter VBM}

To investigate whether individual differences in fornix gross morphology are related to recognition memory performance, VBM (Ashburner and Friston, 2000) of white matter was performed on the T1-weighted structural images. For statistical analyses, the same GLMs that were implemented for TBSS were used, with a statistical threshold of $p<0.005$ uncorrected, cluster size $\geq 5$ voxels.

\section{Results}

\section{Behavioral performance}

Table 1 summarizes the behavioral data from all tasks. To compare performance across the two recognition tasks (Fig. 1), paired $t$ tests were conducted between the various measures of recognition. There was a significant difference between scene and object familiarity $\left(t_{(24)}=2.23, p=0.036\right)$ as derived using the DPSD model, with only a trend toward significance between scene and object recollection $\left(t_{(24)}=1.88, p=0.073\right)$. Considering the UVSD model measures, there was a significant difference between object and scene $z$-ROC slope, and object and scene sensitivity (both $t_{(24)}>2.5, p<0.02$ ). 
Table 1. Behavioral performance across all tasks

\begin{tabular}{|c|c|c|c|c|}
\hline Task & Measure & Mean & SD & Range \\
\hline \multirow[t]{4}{*}{ Scene ROC } & ro & 0.37 & 0.16 & 0.10 to 0.72 \\
\hline & $d^{\prime}$ & 0.65 & 0.40 & -0.04 to 1.49 \\
\hline & z-ROC slope & 0.72 & 0.12 & 0.43 to 0.98 \\
\hline & $\mathrm{Da}$ & 1.17 & 0.46 & 0.56 to 2.18 \\
\hline \multirow[t]{4}{*}{ Object ROC } & ro & 0.42 & 0.18 & 0.12 to 0.72 \\
\hline & $d^{\prime}$ & 0.79 & 0.51 & -0.51 to 1.63 \\
\hline & z-ROC slope & 0.84 & 0.19 & 0.51 to 1.15 \\
\hline & $\mathrm{Da}$ & 1.34 & 0.39 & 0.74 to 2.55 \\
\hline Color oddity & Proportion correct & 0.78 & 0.10 & 0.60 to 0.94 \\
\hline \multirow[t]{3}{*}{ Motor attention } & Neutral response times/ms & 8095 & 1255 & 6204 to 11,536 \\
\hline & Valid response times/ms & 7431 & 1279 & 5111 to 9720 \\
\hline & Invalid response times/ms & 8395 & 1357 & 6173 to 11,166 \\
\hline
\end{tabular}

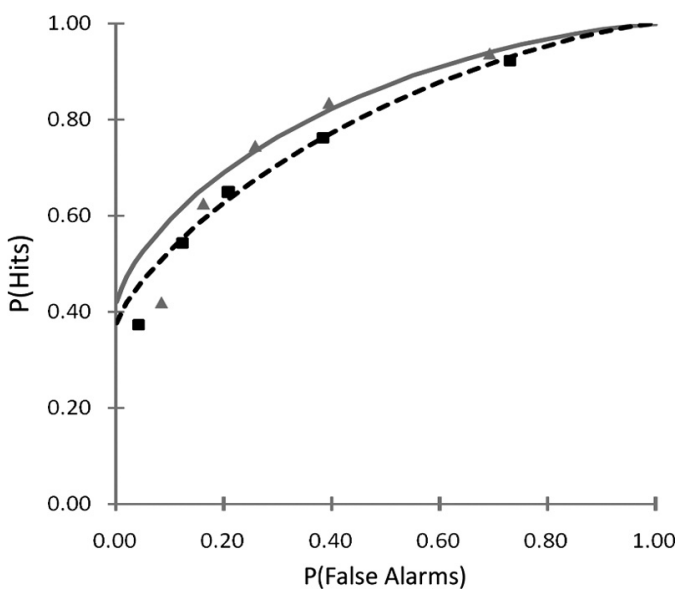

Figure 1. Average ROC curves for the scene (dashed line/squares) and object (solid line/ triangles) recognition tasks.

To assess whether individuals that performed better on one recognition task also performed better on the other, correlational analyses were conducted across the various recognition measures from the scene and object tasks. Both recollection and familiarity estimates correlated significantly (both $r=$ $0.78 ; p \leq 0.0001$ ), with no significant correlation between recollection and familiarity for either scene or object tasks (both $r \leq 0.3, p>0.1$ ). There was a significant correlation between scene and object sensitivity $(r=0.75, p \leq 0.0001)$ but not between scene and object $z$-ROC slope $(r=0.017, p=$ $0.94)$. There was also no correlation between $z$-ROC slope and Da for either scenes or objects (both $r<0.3, p>0.1$ ).

\section{Imaging results \\ TBSS}

Given our a priori hypotheses, only results pertaining to the fornix are reported below. Findings outside of this tract are reported in the supplemental material (available at www.jneurosci.org as supplemental material).

DPSD model. Compared with familiarity, recollection [ (scene + object recollection $)-$ (scene + object familiarity)] was associated with two significant clusters in the left fornix tail (cluster 1 $\max t=5.44 ; x=-18, y=-30, z=5$; cluster $2 \max t=3.07$; $x=-24, y=-33, z=5$ ) (Fig. 2A). FA was extracted at the most significant voxel to illustrate a significant correlation between scene $(r=0.75, p \leq 0.0001)$ and object $(r=0.54, p=0.006)$ recollection and FA, but not between either familiarity estimate and FA (both $r<0.1, p>0.5$ ) (Fig. $2 B, C$ ). The reverse comparison $[($ scene + object familiarity $)-($ scene + object recollec- tion)] revealed no significant findings, suggesting that there was no region within the fornix at which there was a significant relationship between familiarity and FA.

The region in the fornix tail where the two significant clusters were found can be problematic to study with TBSS since narrowness and geometry of the tract can cause relatively low FA values in individual fornix voxels. Consequently, when individual subject maximal FA values are projected onto the mean FA skeleton, values can be projected from nearby fiber tracts rather than the fornix itself. To assess this in our data the significant TBSS clusters were deprojected back into each subject's native space. Although most voxels from within these clusters deprojected back into the fornix for all subjects, a small proportion of cluster voxels were found to have been erroneously projected from the internal capsule in 16 participants. To test whether our correlations still stood without the contribution made by these voxels, we manually removed these voxels from individual subject FA skeletons and calculated mean FA across remaining voxels. After this editing, we still found a significant correlation between FA and scene recollection $(r=0.51 ; p=0.01)$ and object recollection $(r=0.43$; $p=0.03$ ) but not between FA and object or scene familiarity (both $r>0.1, p>0.4$ ).

The possibility that scene and object memory may correlate differentially with fornix FA was also explored. The contrast "(scene recollection + familiarity) - (object recollection $+\mathrm{fa}-$ miliarity)" revealed a significant cluster in the fornix tail ( $\max t=$ 3.36; $x=-18, y=-31, z=6$ ). This cluster is almost identical to that observed in association with recollection and as shown in Figure $2 B$, is driven by a higher correlation between scene recollection and FA than that between object recollection and FA in this region (with no significant relationship between FA and scene or object familiarity). To confirm this, the contrast "scene recollection - object recollection" identified the same cluster ( $\max t=3.46, x=-18, y=-31, z=6)$. No suprathreshold clusters emerged for object memory [i.e., (object recollection + familiarity) - (scene recollection + familiarity)].

UVSD model. Neither measure of performance, slope nor sensitivity parameters, was associated with any suprathreshold clusters of correlation.

Control tasks. No behavioral measures from either the color oddity or attention task were associated with significant clusters of correlation, supporting the notion that the fornix is particularly important for mnemonic processing.

\section{Individual subject tractography and FA extraction}

DPSD model. Confirming the TBSS findings, mean FA of the fornix tail correlated significantly with scene recollection $(r=$ $0.43 ; p=0.034)$, with a trend toward significance for object recollection $(r=0.38 ; p=0.060)$ (Fig. $2 C, D)$. There was also a trend toward a significant correlation between fornix body mean FA and scene recollection $(r=0.37, p=0.071)$ but not between fornix body or column mean FA and scene or object recollection (all $r<0.3, p>0.1$ ). When the fornix was considered as a whole, there was still a significant correlation between mean FA and scene recollection $(r=0.43 ; p=0.034)$ but not object recollection $(r=0.32 ; p=0.12)$. There were no significant correlations between mean FA in the fornix tail, body, or column or the entire fornix and either scene or object familiarity (all $r<0.3$, $p>0.1)$.

UVSD model. Other than a trend toward significance between scene sensitivity and fornix column mean FA $(r=0.38, p=$ 0.062 ), all other UVSD estimates for the scene (all $r<0.4, p>$ 
0.09) and object (all $r<0.3, p>0.2$ ) tasks did not correlate with mean FA in the fornix tail, body, or column or the entire fornix.

Control tasks. No behavioral measures from either control task correlated significantly with mean FA in the fornix tail, body, or column or entire fornix (all $r<$ $0.3, p>0.1)$.

White matter voxel-based morphometry There were no significant correlations between gross morphometry of the fornix and any of the recognition memory estimates or control task behavioral measures.

\section{Discussion}

Using DWI, we have succeeded in studying the involvement of the fornix in recognition memory. Individual differences in the white matter microstructure of this tract, particularly in the tail region, were found to reflect variation in recollection but not familiarity memory on two recognition tasks when behavior was modeled by a dual-process model of recognition memory (DPSD) (Yonelinas, 1994). There was no significant relationship between fornix microstructure and memory performance when behavior was modeled by a unitary model of recognition memory (UVSD) (Wixted, 2007), nor performance on two non-mnemonic tasks (color discrimination and attention).

The current study supports the idea that there are distinct MTL-medial diencephalon pathways that mediate different memory processes (Aggleton and Brown, 1999). As the fornix is comprised predominantly of hippocampal connections, our data underline the suggestion that the hippocampal-diencephalon pathway does not play a general role in recognition memory but is particularly important for recollection (Aggleton and Brown, 1999; Eichenbaum et al., 2007). Our findings are consistent with reports of a role for the hippocampus in recollection but not familiarity memory (Yonelinas et al., 2002; Davachi et al., 2003; Ranganath et al., 2004; Aggleton et al., 2005) and disagree with the suggestion that memory strength is a more critical determinant of MTL involvement in recognition (Squire et al., 2007). The present data also support work that has associated fornix and medial diencephalic damage in human amnesic patients with disproportionate impairment in recollection (Tsivilis et al., 2008; Vann et al., 2009) but, importantly, adds to these studies by identifying, for the first time, the involvement of the fornix without potential influences from neighboring structures (e.g., mammillary bodies) that are commonly also damaged in amnesic cases.

Interestingly, we found an effect of stimulus type, with a more significant relationship between fornix FA and scene recollection memory than that between fornix FA and object recollection. This is not surprising since the hippocampus is critical for spatial cognition (O'Keefe and Nadel, 1978). Indeed, recent work has suggested that stimulus type (objects/scenes) may be a more important determinant of MTL involvement than process type (recollection/familiarity) in recognition memory, with reports of impaired scene but intact face recognition memory in patients with hippocampal damage (Cipolotti et al., 2006; Taylor et al., 2007; Bird et al., 2008). At first glance, our findings do not fit entirely with this view: there was no significant relationship between scene familiarity memory and fornix FA, and better object recollection was associated with improved fornix microstructure. It is possible, however, that our results can be reconciled to a stimulus-dependent view by considering the relationship between recollection and spatial cognition. Recollection is defined by the remembering of contextual information and as such, may often be associated with greater spatial processing, regardless of the stimulus material presented. Likewise, a familiarity trace for a spatial scene may only require minimal spatial processing of that scene and thus, may occur independently from the hippocampus. The relationship between stimulus type and mnemonic process is undoubtedly complex (Diana et al., 2007; Bird and Burgess, 2008) and requires much investigation if functional specialization within the MTL is to be fully understood. 
Due to the orientation and narrow dimensions of the fornix, the use of DWI and TBSS to study this tract can be prone to partial volume errors affecting the estimation of FA at a voxel level. We conducted a number of complementary analyses to ensure that any significant findings were related to FA, and thus white matter microstructure, in the fornix only. First, any significant TBSS clusters at a group level were deprojected back into individual subject space so that any projection errors from neighboring tracts could be identified and their contribution removed. Second, probabilistic tractography was used to identify each participant's fornix, from which mean FA was extracted and correlated with the acquired behavioral measures. Finally, white matter VBM was conducted to investigate whether any significant TBSS results could be accounted for, at least partially, by individual differences in gross fornix morphology. Critically, the findings from all three approaches were highly consistent. Both TBSS and the extraction of mean FA from individual fornices implicated FA in the fornix tail as being significantly related to recollection but not familiarity memory. Moreover, differences in fornix morphology were not found to be related to recollection memory performance.

The current results support a previous study that reported preliminary evidence for a significant correlation between fornix FA and performance on a standard memory task (Doors and People Test) in 11 healthy participants (Nestor et al., 2007). Critically, our findings build considerably upon this by identifying the specific mnemonic processes that the fornix may be important for in a large group of participants.

As with most correlational analyses, the causal direction of our findings is unclear. It is possible that the inherent existence of better fornix microstructure is underlying improved recollection memory in some participants. Conversely, it is feasible that the utilization and improvement of recollection in individuals over time may lead to an enhancement in fornix microstructure. Regardless of this, the biological interpretation of our data requires consideration. FA is believed to reflect axon density, myelination, and diameter (Beaulieu, 2002). The degradation of white matter microstructure, particularly myelination, with aging has been suggested to contribute to cognitive decline by reducing axonal conduction speeds and disrupting the function of neuronal networks (Peters, 2002). It is possible, therefore, that improved fornix microstructure in neurologically healthy individuals may lead to more efficient transfer of information between the hippocampus and medial diencephalon and subsequently, other structures involved in recollection (Fields, 2008). This, in turn, may lead to enhanced encoding and retrieval of information across neural circuits, resulting in better recollective memory.

Although FA in the fornix tail was particularly implicated in this study as correlating with recollection, there was a significant relationship between mean FA across the entire fornix and scene recollection. Consequently, it is unlikely that only white matter microstructure in the fornix tail is critical for recollection. Instead, FA in the fornix tail varied to a greater extent in our study and thus, surpassed the statistical threshold we adopted in our TBSS analyses. The tail section is perhaps one segment of the fornix that contains the highest density of fibers due to its relatively narrow dimensions (e.g., compared with the fornix body). Since FA is sensitive to fiber complexity and compression, it is conceivable, therefore, that FA values in the fornix tail are more likely to fluctuate to a larger degree across individuals.

To summarize, we have used DWI to demonstrate that the involvement of the fornix in memory tasks is significantly greater for recollection than for familiarity. This finding lends support to the notion that distinct recognition processes are subserved by different MTL and diencephalic structures.

\section{References}

Aggleton JP (2008) EPS Mid-Career Award 2006. Understanding anterograde amnesia: disconnections and hidden lesions. Q J Exp Psychol (Colchester) 61:1441-1471.

Aggleton JP, Brown MW (1999) Episodic memory, amnesia and the hippocampal-anterior thalamic axis. Behav Brain Sci 22:425-444; discussion 444-489.

Aggleton JP, McMackin D, Carpenter K, Hornak J, Kapur N, Halpin S, Wiles CM, Kamel H, Brennan P, Carton S, Gaffan D (2000) Differential cognitive effects of colloid cysts in the third ventricle that spare or compromise the fornix. Brain 123:800-815.

Aggleton JP, Vann SD, Denby C, Dix S, Mayes AR, Roberts N, Yonelinas AP (2005) Sparing of the familiarity component of recognition memory in a patient with hippocampal pathology. Neuropsychologia 43:1810-1823.

Ashburner J, Friston KJ (2000) Voxel-based morphometry-the methods. Neuroimage 11:805-821.

Barense MD, Gaffan D, Graham KS (2007) The human medial temporal lobe processes online representations of complex objects. Neuropsychologia 45:2963-2974.

Basser PJ, Mattiello J, LeBihan D (1994) Estimation of the effective selfdiffusion tensor from the NMR spin echo. J Magn Reson B 103:247-254.

Beaulieu C (2002) The basis of anisotropic water diffusion in the nervous system-a technical review. NMR Biomed 15:435-455.

Behrens TE, Woolrich MW, Jenkinson M, Johansen-Berg H, Nunes RG, Clare S, Matthews PM, Brady JM, Smith SM (2003) Characterization and propagation of uncertainty in diffusion-weighted MR imaging. Magn Reson Med 50:1077-1088.

Bilir E, Craven W, Hugg J, Gilliam F, Martin R, Faught E, Kuzniecky R (1998) Volumetric MRI of the limbic system: anatomic determinants. Neuroradiology 40:138-144.

Bird CM, Burgess N (2008) The hippocampus and memory: insights from spatial processing. Nat Rev Neurosci 9:182-194.

Bird CM, Vargha-Khadem F, Burgess N (2008) Impaired memory for scenes but not faces in developmental hippocampal amnesia: a case study. Neuropsychologia 46:1050-1059.

Cipolotti L, Bird C, Good T, Macmanus D, Rudge P, Shallice T (2006) Recollection and familiarity in dense hippocampal amnesia: a case study. Neuropsychologia 44:489-506.

Davachi L, Mitchell JP, Wagner AD (2003) Multiple routes to memory: distinct medial temporal lobe processes build item and source memories. Proc Natl Acad Sci U S A 100:2157-2162.

D’Esposito M, Verfaellie M, Alexander MP, Katz DI (1995) Amnesia following traumatic bilateral fornix transection. Neurology 45:1546-1550.

Diana RA, Yonelinas AP, Ranganath C (2007) Imaging recollection and familiarity in the medial temporal lobe: a three-component model. Trends Cogn Sci 11:379-386.

Eichenbaum H, Yonelinas AP, Ranganath C (2007) The medial temporal lobe and recognition memory. Annu Rev Neurosci 30:123-152.

Fields RD (2008) White matter in learning, cognition and psychiatric disorders. Trends Neurosci 31:361-370.

Gaffan EA, Gaffan D, Hodges JR (1991) Amnesia following damage to the left fornix and to other sites. A comparative study. Brain 114:1297-1313.

Garcia-Bengochea F, Friedman WA (1987) Persistent memory loss following section of the anterior fornix in humans. A historical review. Surg Neurol 27:361-364.

Hodges JR, Carpenter K (1991) Anterograde amnesia with fornix damage following removal of IIIrd ventricle colloid cyst. J Neurol Neurosurg Psychiatry 54:633-638.

Macmillan NA, Creelman CD (2004) Detection theory: a user's guide, Ed 2. Mahwah, NJ: Erlbaum.

McMackin D, Cockburn J, Anslow P, Gaffan D (1995) Correlation of fornix damage with memory impairment in six cases of colloid cyst removal. Acta Neurochir (Wien) 135:12-18.

Nestor PG, Kubicki M, Kuroki N, Gurrera RJ, Niznikiewicz M, Shenton ME, McCarley RW (2007) Episodic memory and neuroimaging of hippocampus and fornix in chronic schizophrenia. Psychiatry Res 155:21-28.

O’Keefe J, Nadel L (1978) The hippocampus as a cognitive map. Oxford: Clarendon. 
Peters A (2002) The effects of normal aging on myelin and nerve fibers: a review. J Neurocytol 31:581-593.

Poreh A, Winocur G, Moscovitch M, Backon M, Goshen E, Ram Z, Feldman Z (2006) Anterograde and retrograde amnesia in a person with bilateral fornix lesions following removal of a colloid cyst. Neuropsychologia 44:2241-2248.

Ranganath C, Yonelinas AP, Cohen MX, Dy CJ, Tom SM, D’Esposito M (2004) Dissociable correlates of recollection and familiarity within the medial temporal lobes. Neuropsychologia 42:2-13.

Rushworth MF, Ellison A, Walsh V (2001) Complementary localization and lateralization of orienting and motor attention. Nat Neurosci 4:656-661.

Smith SM, Jenkinson M, Johansen-Berg H, Rueckert D, Nichols TE, Mackay CE, Watkins KE, Ciccarelli O, Cader MZ, Matthews PM, Behrens TE (2006) Tract-based spatial statistics: voxelwise analysis of multi-subject diffusion data. Neuroimage 31:1487-1505.

Squire LR, Wixted JT, Clark RE (2007) Recognition memory and the medial temporal lobe: a new perspective. Nat Rev Neurosci 8:872-883.

Taylor KJ, Henson RN, Graham KS (2007) Recognition memory for faces and scenes in amnesia: dissociable roles of medial temporal lobe structures. Neuropsychologia 45:2428-2438.

Tsivilis D, Vann SD, Denby C, Roberts N, Mayes AR, Montaldi D, Aggleton JP (2008) A disproportionate role for the fornix and mammillary bodies in recall versus recognition memory. Nat Neurosci 11:834-842.

Vann SD, Tsivilis D, Denby CE, Quamme JR, Yonelinas AP, Aggleton JP, Montaldi D, Mayes AR (2009) Impaired recollection but spared familiarity in patients with extended hippocampal system damage revealed by 3 convergent methods. Proc Natl Acad Sci U S A 106:5442-5447.

Wixted JT (2007) Dual-process theory and signal-detection theory of recognition memory. Psychol Rev 114:152-176.

Yonelinas AP (1994) Receiver-operating characteristics in recognition memory: evidence for a dual-process model. J Exp Psychol Learn Mem Cogn 20:1341-1354.

Yonelinas AP, Kroll NE, Quamme JR, Lazzara MM, Sauvé MJ, Widaman KF, Knight RT (2002) Effects of extensive temporal lobe damage or mild hypoxia on recollection and familiarity. Nat Neurosci 5:12361241 . 